\title{
Low-molecular-weight lipoprotein (a) and low relative lymphocyte concentration are significant and independent risk factors for coronary heart disease in patients with type 2 diabetes mellitus: Lp(a) phenotype, lymphocyte, and coronary heart disease
}

Tatsuya Suzuki ${ }^{*}$, Shoko Futami-Suda ${ }^{1}$, Yoshimasa Igari ${ }^{1}$, Kentaro Watanabe ${ }^{1}$, Motoshi Ouchi ${ }^{1}$, Kazunari Suzuki $^{1}$, Ken-ichi Sekimizu', Yoshiaki Kigawa ${ }^{1}$, Hiroshi Nakano ${ }^{1}$ and Kenzo Oba ${ }^{1}$

\begin{abstract}
Background: The aim of the present prospective study was to examine whether lipoprotein (a) [Lp(a)] phenotypes and/or low relative lymphocyte concentration (LRLC) are independently associated with coronary heart disease (CHD) in patients with type 2 diabetes mellitus (T2DM).

Methods: Serum Lp(a) concentration, Lp(a) phenotypes, and RLC were analyzed in 214 subjects. Lp(a) phenotypes were classified into 7 subtypes according to sodium dodecyl sulfate-agarose gel electrophoresis by Western blotting. Subjects were assigned to the low-molecular-weight (LMW (number of KIV repeats: 11-22) ) and high-molecular-weight (HMW( number of KIV repeats: $>22$ )) Lp(a) groups according to Lp(a) phenotype and to the LRLC (RLC: $<20.3 \%)$ and normal RLC (NRLC; RLC: $\geq 20.3 \%$ ) groups according to RLC. A CHD event was defined as the occurrence of angina pectoris or myocardial infarction during the follow-up period.
\end{abstract}

Results: During the follow-up period, 30 cases of CHD events were verified. Neutrophil count showed no correlation with CHD, while relative neutrophil concentration and RLC showed positive and negative correlations, respectively, with CHD. The Cox proportional hazard model analysis revealed the following hazard ratios adjusted for LMW Lp(a), LRLC, and LMW Lp(a) + LRLC: $(4.31 ; 95 \%$ confidence interval [CI], 1.99-9.32; $P<0.01,3.621 ; 95 \% \mathrm{Cl}, 1.50-8.75 ; P<0.05$, and $7.15 ; 95 \% \mathrm{Cl}, 2.17-23.56 ; P<0.01$, respectively).

Conclusions: Our results suggest that both LMW Lp(a) and LRLC are significant and independent risk factors for CHD and that the combination thereof more strongly predicts CHD in patients with T2DM.

Keywords: Lipoprotein (a) phenotype, Relative lymphocyte concentration, Coronary heart disease

\footnotetext{
* Correspondence: t-suzuki@nms.ac.jp

'Department of Internal Medicine (Divisions of Cardiology, Hepatology, Geriatric Medicine, and Integrated Medicine), Nippon Medical School, 1-1-5, Sendagi, Bunkyo-ku, Tokyo 113-8603, Japan

Full list of author information is available at the end of the article
} 


\section{Background}

Recent studies have reported that diabetes mellitus may correlate with coronary heart disease (CHD) [1,2]. However, conventional risk factors for CHD in previous studies do not account for all cases of CHD. Furthermore, epidemiologic studies have consistently shown a significant relationship between white blood cell (WBC) counts and the onset of CHD [3,4]. Similarly to WBC counts, relative lymphocyte concentration (RLC) is also a strong predictor of CHD [5-7]. Inflammatory markers are elevated in acute coronary syndrome (ACS), and ACS is characterized by unstable atherosclerotic plaque that is activated by a systemic inflammatory reaction [8]. Furthermore, low RLC (LRLC) is related to system inflammation [9]. In addition, recent studies have focused on lymphocytes (especially, RLC) and have reported the value of this clinical parameter $[10,11]$.

Lipoprotein (a) [Lp(a)], a plasma complex composed of apolipoprotein (a) [apo(a)] covalently linked to apo B-100 [12], is an important risk factor for CHD [13,14]. Serum $\mathrm{Lp}(\mathrm{a})$ is a genetically determined independent risk factor, with a presumed variation range of $\leq 10 \%$ [15]. Furthermore, a recent study has shown that apo(a) has a high degree of genetic polymorphism and that this polymorphism may have a predictive value greater than serum $L p(a)$ concentration because $\mathrm{Lp}(\mathrm{a})$ phenotypes possess a genetic trait not influenced by environmental factors as does serum Lp(a) concentration [15]. However, this factor alone does not fully account for the onset of CHD.

To the best of our knowledge, no study in general outpatients with type 2 diabetes mellitus (T2DM) has examined the relationship between apo(a) phenotypes and RLC. Therefore, we examined the effects between each $\mathrm{Lp}(\mathrm{a})$ phenotype and RLC on the onset of CHD in patients with T2DM during the follow-up period.

\section{Methods}

\section{Study participants}

The present prospective study enrolled 214 Japanese outpatients with T2DM (115 men and 99 women) who were referred to our hospital since 1995. All subjects provided informed consent. The present study was designed in compliance with the ethic regulations set out by the Helsinki Declaration. They underwent a standardized interview and physical examination. Electrocardiography was performed at baseline. In the present study, CHD was defined as the new onset of angina pectoris or myocardial infarction during the follow-up period. The attending physician and the cardiologist followed the diagnostic criteria to diagnose angina pectoris as an endpoint.

Myocardial infarction was diagnosed by the presence of at least 2 of the following 3 criteria: 1 ) a clinical history of central chest pressure, pain, or tightness lasting for 30 minutes or longer; 2) an ST segment elevation greater than $0.1 \mathrm{mV}$ in at least 1 standard or 2 precordial leads, an ST segment depression greater than $0.1 \mathrm{mV}$ in at least 2 leads, abnormal $\mathrm{Q}$ wave, or $\mathrm{T}$ wave inversion in at least 2 leads; and 3) an increase in serum creatine kinase concentration to twofold the upper limit of normal. Angina pectoris was diagnosed based on the repeated episodes of chest pain during an effort that usually disappeared shortly after the cessation of the effort or the sublingual administration of nitroglycerin. Angina pectoris was defined to present an ST segment depression greater than $1 \mathrm{~mm}$ on electrocardiograms obtained during chest pain. Patients with T2DM were diagnosed according to the World Health Organi-zation criteria. Patients with hypertension were defined as persons who had a systolic blood pressure (SBP) of $140 \mathrm{mmHg}$ or higher or a diastolic blood pressure (DBP) of $90 \mathrm{mmHg}$ or higher, or who were concurrently receiving antihypertensive drugs. Patients with dyslipidemia were defined as persons who had a serum total cholesterol concentration of $220 \mathrm{mg} / \mathrm{dL}$ or higher, a serum low-density lipoprotein cholesterol (LDL-C) concentration of $140 \mathrm{mg} /$ dL or higher, a serum triglyceride concentration of $150 \mathrm{mg} /$ $\mathrm{dL}$ or higher, or a serum high-density lipoprotein cholesterol (HDL-C) concentration of $40 \mathrm{mg} / \mathrm{dL}$ or lower, or who were concurrently receiving lipid-lowering drugs. Regarding chronic kidney disease (CKD), the estimated glomerular filtration rate was calculated as per the study of Matsuo et al. based on the Japanese Society of Nephrology's guidelines for CKD, and its grades were determined [16]. HbA1c (\%) is given as National Glycohemoglobin Standardization Program (NGSP) equivalent values (\%), which were calculated using the following formula [17]: HbA1c $(\%)=\mathrm{HbA1c}$ (Japan Diabetes Society; \%) $+0.4 \%$. LDL-C was calculated using Friedewald's formula [18].

\section{Anthropometric measurements}

The body mass index was calculated as weight (in kilograms) divided by the square of height (in meters). At screening, subjects who had never smoked and ex-smokers were classified as nonsmokers, while those who were currently smoking were categorized as current smokers.

Exclusion criteria included a history of liver cirrhosis, tuberculosis, patients receiving glucocorticoids over the previous 6 weeks for rheumatoid arthritis, neoplasms, and Basedow disease. To the extent possible, patients with heart failure who had physical activity restrictions (NYHA II or greater) were excluded.

\section{Assessment of $\operatorname{Lp}(\mathrm{a})$ phenotypes and their subgroups}

Serum Lp(a) concentrations were determined according to the latex agglutination (LA) method. Within-run CVs ranged from 1.9 to $2.1 \%$ and between-run CVs from 2.7 to 3.9\% (Lp(a) Latex (Sekisui Medical, Tokyo, Japan). Apo(a) phenotyping was performed according to sodium dodecyl sulfate-agarose gel electrophoresis by Western blotting. Apo(a) phenotypes were classified into 7 subtypes [F, B, 
S1, S2, S3, S4, and O (null)] [19]. The Lp(a) gene is characterized by extensive size polymorphism caused by a variable number of Kringle IV-2 (KIV-2) repeats that are transcribed and translated into protein isoforms of different sizes [20]. Kronenberg et al. considered 11-22 and $>22$ KIV repeats as LMW and HMW, respectively. A conventional cutoff margin was established between $640 \mathrm{Kda}$ and $655 \mathrm{Kda}$ to group LMW and HMW apo(a) phenotypes. The LMW group included patients having at least one apo(a) with 1122 KIV repeats; the HMW group comprised patients having only isoforms with more than $22 \mathrm{KIV}$ repeats [20]. At the commencement of the follow-up period, subjects were assigned to either of the following two groups according to their Lp(a) phenotypes: the LMW (F, B, S1, and S2) Lp(a) group (number of KIV repeats: 11-22) and the HMW (S3, S4, and O) Lp(a) group (number of KIV repeats: $>22$ ) as previously described $[21,22]$. When the subject had a double band, the faster band was used to express the phenotype [23].

\section{Biochemical measurements}

After an overnight fast, blood samples were collected to measure plasma blood glucose levels and serum lipid concentrations. Plasma glucose levels were measured according to the glucose oxidase method. Serum concentrations of total cholesterol, HDL-C, and triglyceride were enzymatically measured with an automatic analyzer. Serum $\mathrm{C}$-reactive protein (CRP) levels were measured by latex agglutination nephelometric immunoassay (LZ test 'Eiken' CRP-HG; Eiken Kagaku Co. Ltd, Tokyo, Japan) and with both intra- and interassay coefficients of $<1.69 \%$. Blood pressures were measured by the physician who used a standard mercury sphygmomanometer, with the subject in the sitting position after at least a 5-minute rest.

Complete blood counts were measured with an automated cell counter (SE-9000, Sysmex, Corporation, Kobe, Japan) according to the standard techniques. Correlations with the manual method for WBC differentials showed good results (neutrophil \%, r $=0.942$; lymphocyte \%, $r=0.937$ ) [24]. The coefficient of variation for repeated measurements of samples from hospitalized patients was maintained at $2.5 \%$. RLC was defined as (total number of lymphocytes/total number of leukocytes) $\times 100$. The normal range of RLC was 20.3 to $50.0 \%$, as defined by the central 95th percentile in a population of healthy subjects [25]. Furthermore, LRLC was defined to present an RLC of $<20.3 \%$ based on previously reported reference values [26]. Subjects were assigned to two groups according to RLC: the low RLC (LRLC; RLC: < 20.3\%) group; and the normal RLC (NRLC; RLC: $\geq 20.3 \%$ ) group.

\section{Follow-up}

Based on previous studies [10,11,27], patients were followed up for about 7 years as a sufficient follow-up period. Information was also obtained for patients who no longer underwent medical care at the hospital. Follow-up data were obtained by the review of medical records or by the telephone interview. We contacted the attending physician directly after obtaining patient consent to inquire about the presence or absence of any CHD event. When the new attending physician was not known, we contacted the patient by telephone to inquire about his/her current clinical condition. Regarding patients with CHD events, we attempted to directly contact the current attending physician. Information about 50 patients was obtained in the above manner. No information could be obtained for 11 patients; these patients were considered withdrawals from the study. Follow-up was discontinued for $13 \mathrm{pa}-$ tients who died of cancer $(n=6)$, pneumonia $(n=4)$, or subarachnoid hemorrhage $(n=3)$.

\section{Statistical analyses}

The baseline characteristics of subjects in the two study groups were compared by using Student's $t$-test for continuous variables and the chi-square test for categorical variables. Pearson's correlation coefficients were used to evaluate correlations between CHD and baseline characteristics. Continuous variables were expressed as mean \pm SD. The Cox proportional hazard model was used to investigate the relationship between the onset of $\mathrm{CHD}$ and the following explanatory variables. A $P$-value of $<0.05$ was considered statistically significant. The SPSS software (Statistical Package, version 11.0; SPSS Inc., Chicago, Il, USA) was used for all statistical analyses.

\section{Results}

Baseline characteristics by $L p(a)$ phenotype of subjects in the HMW $\mathrm{Lp}(\mathrm{a})$ group and the LMW $\mathrm{Lp}(\mathrm{a})$ group are shown in Table 1 . Serum Lp(a) was significantly higher $(P<0.001)$ in the LMW Lp(a) group than in the HMW $\mathrm{Lp}(\mathrm{a})$ group. Significant differences were not found between the two study groups with regard to other variables. Furthermore, the medication rate of statins/fibrates and the medication rate of ACE-I/ARBs were significantly higher in the LMW Lp(a) group than in the HMW Lp(a) group ( $P=0.004$ and $P=0.031$, respectively). However, no significant difference was found between the two groups with respect to the medication rates of calcium channel blockers (CCBs), antiplatelets, and the complication rate of peripheral artery disease, stroke, and CKD II/III.

Baseline characteristics by RLC are shown in Additional file 1: Table S1. Neutrophil counts and relative neutrophil concentration were significantly higher, and lymphocyte counts and relative monocyte concentration and relative lymphocyte concentration were significantly lower $(P=0.001, P<0.001, P<0.001, P=0.018$, $P<0.001$, respectively) in the LRLC group than in the NRLC group. Significant differences were not found 
Table 1 Baseline characteristics by lipoprotein (a) [Lp(a)] phenotype

\begin{tabular}{|c|c|c|c|c|}
\hline $\begin{array}{l}\text { Baseline } \\
\text { characteristics }\end{array}$ & $\begin{array}{l}\text { All subjects } \\
(\mathrm{n}=214)\end{array}$ & $\begin{array}{l}\text { HMW Lp(a) (no. of KIV2 repeats: > 22) } \\
\text { group }(n=169)\end{array}$ & $\begin{array}{l}\text { LMW Lp(a) (no. of KIV } 2 \\
\text { repeats: } 11-22) \text { group }(n=45)\end{array}$ & $P$-value \\
\hline Age (years) & $62 \pm 10$ & $61 \pm 10$ & $65 \pm 10$ & 0.068 \\
\hline Gender (male/female) & $105 / 99$ & $93 / 76$ & $22 / 23$ & 0.489 \\
\hline $\begin{array}{l}\text { White blood cells } \\
\left(/ \mathrm{mm}^{3}\right)\end{array}$ & $6,197 \pm 1563$ & $6,134 \pm 1609$ & $6,447 \pm 1362$ & 0.250 \\
\hline Neutrophil $\left(\mathrm{mm}^{3}\right)$ & $3,711 \pm 1223$ & $3,677 \pm 1262$ & $3,838 \pm 1057$ & 0.433 \\
\hline Monocyte $\left(/ \mathrm{mm}^{3}\right)$ & $348 \pm 142$ & $360 \pm 139$ & $399 \pm 152$ & 0.101 \\
\hline Basophil $\left(/ \mathrm{mm}^{3}\right)$ & $37 \pm 46$ & $40 \pm 62$ & $34 \pm 30$ & 0.527 \\
\hline Eosinophil $\left(/ \mathrm{mm}^{3}\right)$ & $173 \pm 132$ & $170 \pm 126$ & $185 \pm 154$ & 0.511 \\
\hline $\begin{array}{l}\text { Lymphocyte } \\
\left(/ \mathrm{mm}^{3}\right)\end{array}$ & $1,889 \pm 617$ & $1,866 \pm 601$ & $1,973 \pm 675$ & 0.305 \\
\hline Neutrophil (\%) & 59.5 & 59.5 & 59.0 & 0.901 \\
\hline Monocyte (\%) & 6.0 & 5.9 & 6.3 & 0.353 \\
\hline Basophil (\%) & 0.6 & 0.6 & 0.5 & 0.302 \\
\hline Eosinophil (\%) & 2.9 & 2.9 & 2.0 & 0.813 \\
\hline Lymphocyte (\%) & 31.0 & 31.0 & 30.8 & 0.884 \\
\hline $\begin{array}{l}\text { Systolic blood } \\
\text { pressure }(\mathrm{mmHg})\end{array}$ & $138 \pm 16$ & $138 \pm 17$ & $138 \pm 15$ & 0.960 \\
\hline $\begin{array}{l}\text { Diastolic blood } \\
\text { pressure }(\mathrm{mmHg})\end{array}$ & $81 \pm 10$ & $81 \pm 10$ & $80 \pm 10$ & 0.676 \\
\hline $\mathrm{Lp}(\mathrm{a})(\mathrm{mg} / \mathrm{dL})$ & $24.1 \pm 26.8$ & $16.2 \pm 13.4$ & $53.5 \pm 40.9$ & $<0.001$ \\
\hline (median: range) & (14.6: 0-221.0) & (13.0: $0-73.3)$ & (41.5: 8-221.0) & \\
\hline $\begin{array}{l}\text { Total cholesterol } \\
(\mathrm{mg} / \mathrm{dL})\end{array}$ & $211 \pm 39$ & $211 \pm 40$ & $213 \pm 35$ & 0.781 \\
\hline $\mathrm{HDL}-\mathrm{C}(\mathrm{mg} / \mathrm{dL})$ & $60 \pm 19$ & $61 \pm 20$ & $58 \pm 17$ & 0.492 \\
\hline Triglyceride (mg/dL) & $134 \pm 92$ & $134 \pm 93$ & $131 \pm 92$ & 0.790 \\
\hline $\mathrm{LDL}-\mathrm{C}(\mathrm{mg} / \mathrm{dL})$ & $125 \pm 31$ & $123 \pm 32$ & $129 \pm 29$ & 0.270 \\
\hline Creatinine (mg/dL) & $1.0 \pm 0.4$ & $1.0 \pm 0.4$ & $0.9 \pm 0.2$ & 0.348 \\
\hline $\begin{array}{l}\text { Fasting plasma } \\
\text { glucose (mg/dL) }\end{array}$ & $166 \pm 65$ & $168 \pm 66$ & $162 \pm 62$ & 0.503 \\
\hline $\mathrm{HbA1c}(\%)$ & $7.9 \pm 1.8$ & $8.0 \pm 1.8$ & $7.6 \pm 1.6$ & 0.222 \\
\hline $\begin{array}{l}\text { Body mass index } \\
\left(\mathrm{kg} / \mathrm{m}^{2}\right)\end{array}$ & $23.3 \pm 4.1$ & $23.3 \pm 4.2$ & $23.6 \pm 4.0$ & 0.665 \\
\hline Statins/fibrates (\%) & 38.8 & 34.1 & 57.8 & 0.004 \\
\hline ACE-I/ARBs (\%) & 22.0 & 18.3 & 35.6 & 0.031 \\
\hline CCBs (\%) & 27.6 & 26.5 & 33.3 & 0.364 \\
\hline Antiplatelets (\%) & 24.3 & 24.5 & 23.3 & 0.862 \\
\hline $\begin{array}{l}\text { Peripheral vascular } \\
\text { disease }\end{array}$ & 5.1 & 5.3 & 4.4 & 0.819 \\
\hline Stroke $(\%)$ & 9.8 & 11.2 & 4.4 & 0.091 \\
\hline CKD I/III (\%) & 93.5 & 92.9 & 95.6 & 0.524 \\
\hline Current smoker (\%) & 27.5 & 26.0 & 32.5 & 0.412 \\
\hline C-reactive protein $(\%)^{\S}$ & 86.9 & 88.1 & 75.0 & 0.342 \\
\hline
\end{tabular}

Values are expressed as mean \pm SD or numeral (\%).

HMW, high-molecular-weight; LMW, low-molecular-weight; §: Proportion of subjects with a C-reactive protein value of $<4.0 \mathrm{mg} / \mathrm{L} ; \mathrm{HDL}-\mathrm{C}$, high-density lipoprotein cholesterol; LDL-C, low-density lipoprotein cholesterol; HbA1C, glycated hemoglobin; ACE-I/ARBs, angiotensin- converting enzyme inhibitors/angiotensin receptor blockers; CCBs, calcium channel blockers; CKD, chronic kidney disease. 
Table 2 Correlation coefficients of lipoprotein (a) [Lp(a)] phenotypes, relative lymphocyt concentration, and coronary heat disease with baseline characteristics during follow-up

\begin{tabular}{|c|c|c|c|}
\hline \multirow[t]{2}{*}{ Baseline characteristics } & \multicolumn{3}{|c|}{ Correlation coefficients } \\
\hline & Lp(a) phenotypes & $\mathrm{RLC}$ & $\mathrm{CHD}$ \\
\hline Age & 0.124 & -0.048 & $0.188^{* *}$ \\
\hline Female gender & 0.050 & 0.073 & 0.030 \\
\hline White blood cells $\left(/ \mathrm{mm}^{3}\right)$ & 0.079 & -0.123 & -0.041 \\
\hline Neutrophil $\left(/ \mathrm{mm}^{3}\right)$ & 0.054 & $-0.380^{* * *}$ & 0.059 \\
\hline Lymphocyte $\left(/ \mathrm{mm}^{3}\right)$ & 0.071 & $0.414^{* * *}$ & $-0.184^{* *}$ \\
\hline Neutrophil (\%) & -0.009 & $-0.557^{* * *}$ & $0.218^{* *}$ \\
\hline Lymphocyte (\%) & -0.010 & $0.568^{* * *}$ & $-0.215^{* *}$ \\
\hline Systolic blood pressure & 0.004 & -0.014 & $0.137^{*}$ \\
\hline Diastolic blood pressure & -0.026 & -0.052 & 0.109 \\
\hline $\operatorname{Lp}(a)$ & $0.568^{* * *}$ & -0.016 & $0.318^{* * *}$ \\
\hline Total cholesterol & 0.023 & 0.117 & -0.081 \\
\hline HDL-C & -0.047 & -0.028 & -0.015 \\
\hline Triglyceride & -0.015 & 0.053 & -0.065 \\
\hline LDL-C & 0.078 & $-0.136^{*}$ & -0.077 \\
\hline Creatinine & -0.064 & -0.008 & -0.038 \\
\hline Fasting plasma glucose & -0.045 & -0.070 & 0.080 \\
\hline $\mathrm{HbA1c}$ & -0.084 & 0.021 & 0.060 \\
\hline Body mass index & 0.031 & 0.080 & 0.043 \\
\hline C-reactive protein $§$ & -0.072 & -0.113 & 0.079 \\
\hline Current smoker & 0.058 & 0.078 & $0.165^{*}$ \\
\hline Statins/fibrates & $0.201^{* *}$ & -0.008 & $0.145^{*}$ \\
\hline ACE-I/ARBs & $0.165^{*}$ & 0.053 & 0.108 \\
\hline Antiplatelets & -0.012 & -0.120 & $0.184^{* *}$ \\
\hline CKD $\|/\|$ & 0.044 & 0.045 & -0.002 \\
\hline
\end{tabular}

Lp(a), lipoprotein (a); HDL-C, high-density lipoprotein cholesterol; LDL-C, low-density lipoprotein.

cholesterol; ACE-I/ARBs, angiotensin-converting enzyme inhibitors/angiotensin receptor blockers; $C K D$, chronic kidney disease $\mathrm{RLC}$, relative lymphocyte concentration; $\mathrm{CHD}$, coronary heart disease. ${ }^{*} P<0.05,{ }^{* *} P<0.01,{ }^{* * *} P<0.001$.

between the two study groups with regard to other variables. However, no significant difference was found between the two groups with respect to the medication rates of calcium channel blockers (CCBs), antiplatelets, and the complication rate of peripheral artery disease, stroke, and CKD II/III.

Correlation coefficients of baseline characteristics with Lp(a) phenotypes, RLC, and CHD are shown in Table 2. No correlation was found between $\mathrm{Lp}(\mathrm{a})$ phenotypes and lymphocyte count and between $\mathrm{Lp}(\mathrm{a})$ phenotypes and RLC. Furthermore, negative correlation was found between RLC and neutrophil count and between RLC and relative neutrophil concentration. Neutrophil count showed no correlation with CHD, while relative neutrophil concentration and RLC showed positive and negative correlations, respectively, with $\mathrm{CHD}$. We performed an ROC analysis on $\mathrm{CHD}$ with respect to WBC, $\mathrm{N}$ (absolute number), Lym (absolute number), \% N, and \% Lym. The AUC value was slightly greater for \% Lym (0.68) than for \% N (0.67) and WBC (0.493). In the present study, therefore, we selected \% Lym although both had a mirror image. No correlation was found between $\operatorname{Lp}(\mathrm{a})$ phenotypes and baseline characteristics except for statins/fibrates and ACE-I/ARBs. Therefore, we regard both $\mathrm{Lp}(\mathrm{a})$ phenotypes and RLC as dependent factors.

Table 3 shows the results from an analysis using the Cox proportional hazard model of survival by $\mathrm{CHD}$ event during follow-up. Age, female gender, CRP, ACE-I/ARBs, SBP, HDL-C, HbA1c, and CKD II/III were selected as variables to adjust the Cox proportional hazards based on Table 1, Table 3, and Additional file 1: Table S1. The Cox proportional hazard model was used to analyze LMW Lp (a), LRLC, and LMW Lp(a) + LRLC. When not adjusted for age, female gender, CRP, ACE-I/ARBs, SBP, HDL-C, HbA1c, and CKD II/III, hazard ratio for LMW Lp(a), LRLC, and LMW Lp(a) + LRLC were 4.45, 3.45, and 11.31, respectively. When adjusted for these variables, hazard ratio for LMW Lp(a), LRLC, and LMW Lp(a) + LRLC were $4.31 ; 95 \%$ confidence interval $[\mathrm{CI}], 1.99-9.32$; $P<0.01,3.62$; $95 \% \mathrm{CI}, 1.50-8.75 ; P<0.05$, and $7.15 ; 95 \%$ CI, 2.17-23.56; $P<0.01$, respectively.

\section{Discussion}

Our results suggest that not only LMW Lp(a) and LRLCwhich are independent predictors for the onset of CHDbut also the combination thereof more strongly predicts CHD. We used the Cox proportional hazard model to analyze LMW Lp(a), LRLC, and LMW Lp(a)+LRLC. When not adjusted, hazard ratio for LMW Lp(a), LRLC, and LMW Lp(a)+LRLC were, 4.45, 3.45, and 11.31 respectively. When adjusted for age, female gender, CRP, ACE-I/ARBs, SBP, HDL-C, HbA1c, and CKD II/III, hazard ratio for LMW Lp(a), LRLC, and LMW Lp(a) + LRLC were, $4.31,3.62$ and 7.15, respectively.

Many previous studies have reported that the incidence of CHD was significantly higher in the elevated $\mathrm{Lp}(\mathrm{a})$ group $[13,14,28]$. Furthermore, $\mathrm{Lp}(\mathrm{a})$ has been reported to compete with plasminogen for the binding sites in a dose-dependent manner. In addition, the risk of developing CHD due to $\mathrm{Lp}(\mathrm{a})$ was attributed to the amount of $\mathrm{Lp}(\mathrm{a})$ that was bound to small-size apo(a) [29]. In the present study, therefore, we investigated how Lp(a) phenotypes, but not serum Lp(a) concentrations, might influence the onset of CHD. We also speculated that every phenotype presents genetic polymorphism as described by Ichinose et al. [30]. Consequently, not only 
Table 3 Cox proportional hazard model of survival by coronary heat disease event during follow-up

\begin{tabular}{lcccc}
\hline Variables & Hazard ratio & $\mathbf{( 9 5 \% ~ C l )}$ & Adjusted hazard ratio & $\mathbf{( 9 5 \% ~ C l )}$ \\
\hline LMW Lp(a) (no. of KIV2 repeats: $11-22)$ & $4.45^{* * *}$ & $(2.18-9.11)$ & $4.31^{\dagger * *}$ & $(1.99-9.32)$ \\
LRLC & $3.45^{* *}$ & $(1.48-8.05)$ & $3.62^{\dagger *}$ & $(1.50-8.75)$ \\
LMW Lp(a) (no. of KIV2 repeats: $11-22)+$ LRLC & $11.31^{* * *}$ & $(3.81-33.24)$ & $7.15^{\dagger * *}$ & $(2.17-23.56)$ \\
\hline
\end{tabular}

†Adjusted for age, female gender, CRP, ACE-I/ARBs, SBP, HDL-C, HbA1C, and CKD II/III.

CRP, C-reactive protein; ACE-I/ARBs, angiotensin-converting enzyme inhibitors/ angiotensin receptor blockers; SBP, systolic blood pressure; HDL-C, high-density lipoprotein cholesterol; HbA1c, glycated hemoglobin; CKD, chronic kidney disease; LMW Lp(a), low-molecular-weight lipoprotein (a); LRLC, low relative lymphocyte concentration.

${ }^{*} P<0.05,{ }^{* *} P<0.01,{ }^{* * *} P<0.001$.

serum $\mathrm{Lp}(\mathrm{a})$ concentrations but also Lp(a) phenotypes were found to be associated with the onset of CHD.

We consider that LRLC is a predictor of CHD based on its diagnostic usefulness according to previous studies $(5,6,7,11)$, as well as on the correlation coefficients of CHD (Table 2) and the results from the ROC analysis about blood cell markers in the present study. Data from experimental work in animals and in vitro data show that leukocytes also play an important role in atherogenesis [31]. Furthermore, previous investigations have demonstrated that LRLC is also an independent predictor for CHD [5,7]. Our results indicated that subjects with LRLC had an adjusted 3.62-fold hazard ratio of developing CHD events during follow-up and that LRLC was an independent predictor for CHD. Our data support at least partially the role of leukocytes in the chronic process of atherosclerosis. Although pathophysiological mechanisms still remain unclear, current evidence suggests that LRLC may be useful for identifying patients with heart disease, e.g., ACS and heart failure. To the best of our knowledge, however, no previous study has examined whether LMW Lp(a) and LRLC, when combined, are strong and independent predictors for the onset of CHD. Our study is the first to address this issue.

According to many previous studies, the causes of lymphocytopenia are regarded as an early marker of stress because the stress-induced increase in cortisol secretion leads to lymphocytopenia [32,33]. The mechanism to induce changes in leukocyte counts probably involves interactions among the nervous, endocrine, and immune systems that differ from the electrical and celldamage mechanisms for electrocardiographic and biochemical markers of infarction [6]. The hypothalamus responds by increasing serum corticotropin-releasing hormone levels, which then causes pulsatile increases in serum cortisol levels.

Not only physiological stress but also mental stress has been speculated to provoke the rupture of the fibrous cap around coronary atherosclerotic plaque [34]. Black $\mathrm{PH}$ et al. hypothesized that stress induces the release of cytokines, which, together with major stress hormones-corticosteroids and catecholamines, induces the production of acute phase proteins in the liver [35] and that various classes of stress can induce the production and release of proinflammatory cytokines which may mediate micro thrombosis.

There are several limitations to our study. First, we enrolled outpatients in a prospective open-label trial, which may potentially involve attribution biases. Furthermore, our study cannot rule out the presence of selection bias due to its nature of being a hospital cohort study. Second, sample size for the onset of CHD may be small. Therefore, a multicenter study enrolling a greater number of patients will be required in the future. Furthermore, highsensitivity CRP could not be measured. There is also a need to determine the number of lymphocyte count measurements by multiple measurements in the future. Third, we did not measure serum cortisol levels to confirm its elevation in association with a reduction in RLC. To the extent possible, patients with heart failure (NYHA II or greater) were excluded. However, ejection fraction (EF) was not determined at baseline. Finally, several Lp(a) phenotypes are considered to exist. A further effort should be made to determine whether other unknown $\mathrm{Lp}(\mathrm{a})$ phenotypes may or may not correlate with the onset of CHD. Moreover, further study using analytical procedures (e.g., pulse-field electrophoresis of unamplified genomic DNA) will elucidate the effects of $L p(a)$ with a specified number of KIV-2 repeats on CHD in the future.

\section{Conclusions}

LMW Lp(a) is an independent risk factor for CHD, and LRLC is also an independent risk factor of CHD. LMW Lp(a) and LRLC, when combined, predict CHD more strongly in patients with T2DM.

\section{Additional file}

Additional file 1: Table S1. Baseline characteristics by relative

lymphocyte concentration.

\section{Abbreviations}

Lp(a): Lipoprotein (a); LMW: Low molecular weight; HMW: High molecular weight; Kringle IV-2: KIV-2; LRLC: Low relative lymphocyte concentration; NRLC: Normal relative lymphocyte concentration; CHD: Coronary heart disease; T2DM: Type 2 diabetes mellitus; LDL-C: Low-density lipoprotein 
cholesterol; HDL-C: High-density lipoprotein cholesterol; ACE-I/ ARBs: Angiotensin-converting enzyme inhibitors/angiotensin receptor blockers; CRP: C-reactive protein; CKD: Chronic kidney disease; SBP: Systolic blood pressure.

\section{Competing interests}

The authors declare that they have no competing interests.

\section{Authors' contributions}

TS and KO contributed to the study conception and design, and writing manuscript. YI, KW and SFS participated in data collection, data analysis. MO, KS and KS contributed to design, conduct, data collection. YK, HN contributed to design, conduct and analysis. All authors read and approved the final manuscript.

\section{Acknowledgements}

The authors thank Dr. Satoshi Sakima for the critical review of the manuscript.

\section{Author details}

'Department of Internal Medicine (Divisions of Cardiology, Hepatology, Geriatric Medicine, and Integrated Medicine), Nippon Medical School, 1-1-5, Sendagi, Bunkyo-ku, Tokyo 113-8603, Japan. ${ }^{2}$ Hanno Geriatric Hospital, Hanno, Saitama, Japan.

\section{Received: 15 December 2012 Accepted: 28 February 2013}

Published: 7 March 2013

\section{References}

1. Rivera NV, et al: Assessment of the 9p21.3 locus in severity of coronary artery disease in the presence and absence of type 2 diabetes. BMC Med Genet 2013, 23:14

2. Enomoto $Y$, et al: Silent coronary artery disease in Japanese patients undergoing carotid artery stenting. J Stroke Cerebrovasc Dis 2013. doi:10.1016/j.jstrokecerebrovasdis.2012.12.013. pii: S1052-3057(12)00440-5. [Epub ahead of print]

3. Kannel WB, et al: White blood cell count and cardiovascular disease. Insights from the Framingham Study. JAMA 1992, 267:1253-1256.

4. Hasegawa T, et al: WBC count, atherosclerosis and coronary risk factors. J Atheroscler Thromb 2002, 2:219-223.

5. Ommen SR, et al: Usefulness of the lymphocyte concentration as a prognostic marker in coronary artery disease. Am J Cardiol 1997 79:812-814

6. Thomson SP, et al: Incremental value of the differential and the rapid creatinine kinase-MB isoenzyme for the early diagnosis of MI. Ann Intern Med 1995, 122:335-341.

7. Zouridakis EG, et al: Usefulness of the blood lymphocyte count in predicting recurrent instability and death in patients with unstable angina pectoris. Am J Cardiol 2000, 86:449-451.

8. Ozer $\mathrm{N}$, et al: Effects of drug-eluting stents on systemic inflammatory response in patients with unstable angina pectoris undergoing percutaneous coronary intervention. Hear Vessel 2008, 23:75-82.

9. Kiank $C$, et al: Seasonal variations in inflammatory responses to sepsis and stress in mice. Crit Care Med 2007, 35:2352-2358.

10. Núñez J, et al: Relationship between low lymphocyte count and major cardiac events in patients with acute chest pain, a non-diagnostic electrocardiogram and normal troponin levels. Atherosclerosis 2009, 206:251-257.

11. Bian C, et al: Predictive value of the relative lymphocyte count in coronary heart disease. Hear Vessel 2010, 25(6):469-473.

12. Utermann G: The mystery of lipoprotein (a). Science 1989, 246:904-910.

13. Rhoads GG, et al: $L p(a)$ lipoprotein as a risk factor for myocardial infarction. JAMA 1986, 256:2540-2544.

14. Suzuki T, et al: Four-year prospective study of the influence of elevated serum lipoprotein (a) concentration on ischemic heart disease and cerebral infarction in elderly patients with type-2 diabetes. Geriatr Gerontol Int 2003, 3:507-513.

15. Boerwinkle E: Genetics of plasma lipoprotein (a) concentrations. Curr Op Lipid 1992, 3:128-136.

16. Matsuo $\mathrm{S}$, et al: Revised equations for estimated GFR from serum creatinine in Japan. Am J Kidney Dis 2009, 53:982-992.
17. The Committee of the Japan Diabetes Society on the diagnostic criteria of diabetes mellitus: Report of the Committee on the classification and diagnostic criteria of diabetes mellitus. Diabetol Int 2010, 1:2-20.

18. Fredewald WT, et al: Estimation of the concentration of low-density lipoprotein cholesterol in plasma, without use of the preparative ultracentrifuge. Clin Chem 1972, 18(6):499-502.

19. Utermann $G$, et al: Lp(a) glycoprotein phenotypes. Inheritance and relation to Lp(a)-lipoprotein concentrations in plasma. J Clin Invest 1987, 80:458-465

20. Kronenberg F, et al: Lipoprotein (a): resurrected by genetics. J Intern Med 2012, 273:6-30.

21. Gazzaruso C, et al: Association of lipoprotein (a) levels and apolipoprotein (a) phenotypes with coronary heart disease in patients with essential hypertension. J Hypertens 1997, 15:227-235

22. Suzuki T, et al: Relation of apolipoprotein (a) phenotypes to Diabetic retinopathy in elderly type 2 diabetes. J Nippon Med Sch 2002, 69:31-38.

23. Hernandez C, et al: Relationship of lipoprotein (a) and its phenotypes with albumin excretion rate in diabetic patients: A multivariate analysis. Nephron 2000, 85:27-33.

24. Research and Development Division, TOA medical Electronics Co. Ltd: The introduction of the Sysmex new automated hematology analyzer SE9000'. Sysmex J 1993, 16:174-191.

25. Matsuno K, et al: Standard Textbook of Laboratory Hematology: Chapter II. (The Japanese Society for Laboratory Hematology, 32-33). Tokyo: Ishiyaku Publishers; 2003. In Japanese.

26. Pierre RV, et al: Comparison of four leukocyte differential methods with the National Committee for Clinical Laboratory Standards (NCCLS) reference method. Am J Clin Pathol 1987, 87:201-209.

27. Baños-González MA, et al: Apo(a) phenotyping and long-term prognosis for coronary artery disease. Clin Biochem 2010, 43:640-644.

28. Bostom AG, et al: A prospective investigation of elevated lipoprotein (a) detected by electrophoresis and cardiovascular disease in women. The Framingham Heart Study. Circulation 1994, 90:1688-1695.

29. Paultre $F$, et al: High levels of $L p(a)$ with a small apo(a) isoform are associated with coronary artery disease in African American and white men. Arterioscler Thromb Vasc Biol 2000, 20:2619-2624.

30. Ichinose A, et al: Detection of polymorphisms in the $5^{\prime}$-flanking region of the gene for apolipoprotein (a). Biochem Biophys Res Commun 1995, 209:372-378.

31. Ross R: Atherosclerosis-an inflammatory disease. N Engl J Med 1999, 340:115-126.

32. Chrousos GP, et al: Mechanisms of physical and emotional stress. (Advances in Experimental Medicine and Biology, 245). New York: Plenum Press; 1989.

33. Naito $Y$, et al: Biphasic changes in hypothalamo-pituitary-adrenal function during the early recovery period after major abdominal surgery. J Clin Endocrinol Metab 1991, 73:111-117.

34. Hemingway $\mathrm{H}$, et al: Evidence based cardiology: psychosocial factors in the aetiology and prognosis of coronary heart disease. Systematic review of prospective cohort studies 1999, 318:1460-1467.

35. Black PH, et al: Stress, inflammation and cardiovascular disease. J Psychosom Res 2002, 52:1-23.

\section{doi:10.1186/1476-511X-12-3}

Cite this article as: Suzuki et al: Low-molecular-weight lipoprotein (a) and low relative lymphocyte concentration are significant and independent risk factors for coronary heart disease in patients with type 2 diabetes mellitus: Lp(a) phenotype, lymphocyte, and coronary heart disease. Lipids in Health and Disease 2013 12:31. 